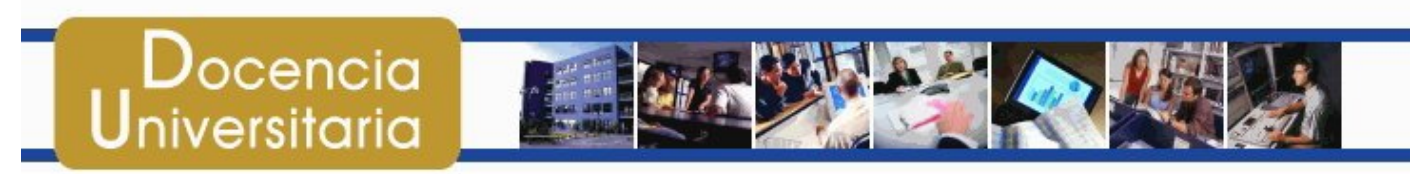

Revista Digital de Investigación en Docencia Universitaria / Añ̃ 4 - Nㅁ- Dic. 2008

\title{
El docente en el desarrollo de la inteligencia emocional: reflexiones y estrategias
}

\author{
Sigrid Buitrón Buitrón \\ Patricia Navarrete Talavera
}

\section{Resumen}

La presente reflexión se centra en la importancia del rol del docente como agente de desarrollo de la inteligencia emocional en sus estudiantes. En las últimas décadas, ha surgido la necesidad de considerar a la educación no solo como un instrumento para el aprendizaje de contenidos y desarrollo de competencias cognitivas, sino también como un espacio que contribuye a la formación integral de los alumnos. Que favorece la construcción y reforzamiento de valores. Que enseña a llevar vidas emocionalmente más saludables. Y que impulsa la convivencia pacífica y armónica.

La educación emocional es entendida como el desarrollo planificado y sistemático de habilidades de autoconocimiento, autocontrol, empatía, comunicación e interrelación. Ha cobrado un papel fundamental y hoy requiere ubicarse de forma transversal en la programación educativa y la práctica docente. En este contexto, se hace indispensable formar maestros “emocionalmente inteligentes”, que puedan cumplir el reto de educar a sus alumnos con un liderazgo democrático. Que, a través de sus experiencias, puedan enseñar a reconocer, controlar y expresar respetuosa y claramente sus emociones. El clima del aula, generado por la actuación del maestro, impactará definitivamente en el aprendizaje de los alumnos.

Palabras clave: inteligencia emocional, educación emocional, docente, formación integral, empatía, competencias personales, competencias sociales. 
En las últimas décadas, la educación viene experimentando un interesante cambio de paradigma. Mientras que en los años noventa se orientaba fundamentalmente al desarrollo cognitivo y la adquisición de conocimientos, actualmente ha reconocido la enorme necesidad de concebir al ser humano como un todo integrado; es decir, por los aspectos cognitivos, afectivos y morales que interactúan permanentemente con el entorno.

En la práctica pedagógica cotidiana, la educación cobra un rol cada vez más necesario e integral. La actual crisis de valores, el aumento de conductas violentas, la falta de disciplina y motivación en los estudiantes, así como el incremento de actitudes discriminatorias e intolerantes, permiten repensar la función de los profesores y replantear los objetivos globales del sistema educativo.

Estas necesidades fueron claramente reflejadas en el informe de Jacques Delors de la UNESCO (1996), que propuso que la educación respondiera a las demandas sociales y fuera un mecanismo de prevención del conflicto humano. Este trabajo manifestaba la enorme preocupación por la incapacidad de las personas de convivir y tolerar las diferencias.

Dicho informe planteó cuatro pilares fundamentales en el desarrollo de la persona:

1. Aprender a conocer: el dominio de las formas o métodos que permiten adquirir, comprender y descubrir conocimiento, y derivar un aporte significativo a la sociedad. Comprende “aprender a aprender” para aprovechar las oportunidades que ofrece la educación a lo largo de la vida.

2. Aprender a hacer: la adquisición de competencias generales que incluyan las destrezas personales necesarias para la productividad (creatividad, trabajo en equipo, toma de decisiones, etc).

3. Aprender a convivir: aprender a descubrir progresivamente a los demás, reconocerse como seres interdependientes de otros, desarrollar la capacidad de resolver conflictos, y respetar los valores de pluralismo, comprensión mutua y paz.

4. Aprender a ser: el desarrollo máximo del potencial humano de la persona y el logro de un pensamiento autónomo.

La educación emocional, entendida como el desarrollo planificado y sistemático de programas educativos que promueven la inteligencia emocional, aparece como una respuesta consecuente y acertada a las necesidades planteadas. Es un complemento 
Aก̃̃ 4- NDי- Dic. 2008

indispensable de desarrollo cognitivo y una herramienta fundamental en la prevención de problemáticas sociales.

\section{La inteligencia emocional}

En el contexto social de los años noventa, surgió el concepto de inteligencia emocional. Esta explicó ciertos aspectos del comportamiento humano asociados a la inteligencia, pero que no respondían exclusivamente al aspecto cognitivo, sino que implicaban también a las emociones.

En 1990, Mayer y Salovey fueron los primeros en acuñar el término de "inteligencia emocional", definiéndola como "la forma de inteligencia social que implica la capacidad de supervisarse a uno mismo y a otros, sus sentimientos y emociones, para diferenciar entre ellos y utilizar esta información para conducir el pensamiento y la acción”.

En otras palabras, la inteligencia emocional se entiende como una habilidad para reconocer, percibir y valorar las propias emociones, así como para regularlas y expresarlas en los momentos adecuados y en las formas pertinentes.

Ya Howard Gardner, en 1983, había planteado la no existencia de una inteligencia única fundamental para el éxito en la vida. Postulaba un amplio espectro de inteligencias con siete variedades claves, entre las que se incluían las inteligencias "intrapersonal” e "interpersonal”. Las tesis de Gardner abrieron, en cierto modo, el desarrollo de una línea que afirmaba la importancia de los elementos afectivos, emocionales y sociales en el desarrollo de la persona, así como en el éxito que pudiera obtener en su interacción con el entorno.

En 1990, Salovey incluyó las inteligencias personales de Gardner en su definición básica de inteligencia emocional. Señaló, asimismo, cinco capacidades fundamentales:

1. Conocer las propias emociones: reconocer un sentimiento mientras ocurre.

2. Manejar las emociones: manejar los sentimientos para que sean los adecuados.

3. Encontrar la motivación: ordenar las emociones al servicio de un objetivo mayor, desarrollando la capacidad de "automotivarse".

4. Reconocer las emociones de los demás: la empatía.

5. Manejar las relaciones: manejar las emociones de los demás dentro del contexto interpersonal y social. Estas habilidades se relacionan al liderazgo y la eficacia interpersonal. 
Aก̃̃ 4- NDי- Dic. 2008

Posteriormente, en 2002, Goleman propuso un modelo de inteligencia emocional que incluyó cuatro aptitudes agrupadas en dos grandes tipos de competencias: la personal y la social. La primera impactaría directamente en el tipo de relación que uno entabla consigo mismo; en la segunda, la competencia social definiría el tipo de vínculos que se establecen con los otros. De forma esquemática, se presenta a continuación el contenido de cada uno de estos dominios:

1. Competencias personales:

a. Conciencia de uno mismo: comprender profundamente las emociones, fortalezas y debilidades, valores y motivaciones. Se sustenta en el desarrollo de tres habilidades: la conciencia emocional, la valoración personal y la confianza en uno mismo.

b. Autogestión: regular los afectos y emociones para actuar con lucidez y claridad, según las demandas de cada situación. En tal sentido, además de la capacidad de regular la expresión de las emociones, se necesitan habilidades como la transparencia, la capacidad de adaptarse a entornos cambiantes y responder con iniciativa y optimismo, y la orientación hacia el logro a través del esfuerzo.

\section{Competencia social:}

a. Conciencia social: ser capaces de comprender los sentimientos ajenos y tomarlos en cuenta durante el proceso de toma de decisiones. Se resalta el rol de la empatía, pero se requiere también del desarrollo de habilidades complementarias, como la facultad de tomar conciencia en la organización de los grupos humanos y la actitud de servicio.

b. Gestión de las relaciones: regular las emociones de las otras personas; inspirarlas y movilizarlas en la dirección adecuada. Para ello, resulta indispensable ser capaz de establecer vínculos auténticos y duraderos, gestionar los conflictos, y trabajar en equipo en favor de los cambios deseables.

\section{La educación emocional y el rol del docente}

Siendo la finalidad de la educación formar estudiantes emocionalmente competentes (capaces de reconocer y manejar sus emociones), y, por lo tanto, de relacionarse con los demás de forma adecuada y pacífica, surge el planteamiento de una educación emocional como forma de implicar al proceso educativo en la búsqueda de este logro.

La educación emocional comprende la promoción del desarrollo de las competencias emocionales antes planteadas, a través de una programación sistemática y progresiva, de acuerdo a las edades de los alumnos que, idealmente, se traslapen con la currícula y 
acompañen al aprendizaje de conocimientos y habilidades. En los colegios, dicha aproximación se hace necesaria desde el nivel muy elemental hasta el egreso de los estudiantes; es decir, en todos los niveles de la educación y en todas las etapas de desarrollo.

Esta intervención, enfocada al desarrollo afectivo y mediada por la educación, ya no debe circunscribirse a actividades aisladas, como las realizadas en la "hora de tutoría". Corresponde, más bien, al acto educativo en sí. Resulta transversal a la práctica docente, por lo que ya no son solo los tutores los encargados de trabajar los temas afectivos, sino también todos los maestros que interactúen con alumnos.

El docente emocionalmente inteligente es, entonces, el encargado de formar y educar al alumno en competencias como el conocimiento de sus propias emociones, el desarrollo del autocontrol y la capacidad de expresar sus sentimientos de forma adecuada a los demás.

Para que el profesor se encuentre preparado para asumir este reto, es necesario, en primer lugar, que piense en su propio desarrollo emocional: solo entonces estará apto para capacitarse y adquirir herramientas metodológicas que le permitan realizar esta labor. Se sabe que es imposible educar afectiva y moralmente a estudiantes si no se cuenta con una estructura de valores clara, además de un cierto dominio de las propias emociones.

El desarrollo de los recursos emocionales del docente debe ser el primer paso para emprender este cambio educativo. El maestro emocionalmente inteligente debe contar con los suficientes recursos emocionales que acompañen el desarrollo afectivo de sus alumnos. Con ello, establece un vínculo saludable y cercano con ellos, comprende sus estados emocionales, y les enseña a conocerse y a resolver los conflictos cotidianos de forma conciliadora y pacífica.

En 2004, Vivas de Chacón realizó una investigación sobre las competencias socioemocionales del docente, con el fin de sugerir un programa de formación del profesorado. A partir de información, recopilada en un conjunto de entrevistas a profundidad, plantea un modelo que identifica cuatro tipos de necesidades que los docentes entrevistados consideran como competencias pendientes de desarrollo:

- Conocimientos sobre inteligencia emocional: sobre las emociones y su relación con los procesos cognitivos, así como el papel que juega la I.E. en la adaptación y establecimiento interpersonal de las personas. 
Aก̃̃ 4- NDי- Dic. 2008

- Habilidades interpersonales: para identificar las emociones de sus alumnos, percibir sus estados de ánimo, escuchar, ser empático, tomar decisiones, resolver conflictos, tener liderazgo y habilidad para dirigir y persuadir, descubrir las fortalezas de los demás, y ser justo y equitativo.

- Habilidades intrapersonales: para controlar, manejar e interpretar las propias emociones, de modo que se pueda reaccionar de forma coherente con ellas.

- Habilidades didácticas para la educación emocional: nuevas competencias profesionales para un modelado más efectivo, para estimular el desarrollo armónico de los alumnos y atender los problemas emocionales; fomenta el desarrollo de competencias didácticas creativas que promuevan escuelas emocionalmente inteligentes, que construyan ambientes propicios y estimulantes para el desarrollo afectivo.

Por otra parte, es necesario recordar que las emociones juegan un papel central en las interacciones sociales, así como en el comportamiento en todos los entornos. Por ejemplo, en el aula, tanto el maestro como los estudiantes experimentan diversas emociones: alegría, cólera, tristeza, miedo, vergüenza, impotencia, satisfacción, aburrimiento, etc. Es decir, el flujo de los afectos es constante y refleja el mundo interno de los estudiantes, así como su estado anímico y su disposición para el aprendizaje.

Un maestro emocionalmente inteligente debe percibir este movimiento afectivo para dirigirlo de forma provechosa para el aprendizaje, basándose en su capacidad interpersonal y liderazgo. Un maestro motivador, conciliador y con buen sentido del humor tendrá un impacto positivo en sus alumnos. Por el contrario, un maestro poco tolerante, rígido y con escaso manejo anímico puede afectar negativamente el clima del aula.

Definitivamente, convertirse en docentes emocionalmente inteligentes es un reto. No solo demanda espacios y tiempos de capacitación y trabajo; también implica un compromiso que trasciende el plano laboral, comprendiendo el plano afectivo y personal. El mundo interno del maestro se mueve: debe crecer como persona, conocerse a sí mismo, y enfrentar sus miedos y conflictos. Esta experiencia, en algunos casos, podría evaluarse como “amenazadora” y ser desarrollada con angustia, alimentando las resistencias. Pese a ello, resulta indispensable dar el primer paso.

En tal sentido, el objetivo de este taller ha sido generar un espacio que favorezca al desarrollo de las aptitudes emocionales en los docentes, condición necesaria para asumir con éxito el rol de modeladores de sus alumnos. Para llevar a cabo este objetivo, se ha fomentado a cada participante a mirarse sí mismo para adquirir herramientas que le permitan evaluarse e identificar el grado de desarrollo de cada una de sus competencias. 


\begin{abstract}
Universitaria
El dacente en el desarrollo de la inteligencia emacional: reflexiones y estrategias

Аก̃̃ 4- Nㅣ- Dic. 2008

Posteriormente, deberá focalizar la mirada sobre aquel otro protagonista del aula: el alumno. Empleando diversos instrumentos, se logrará así una comprensión más profunda de los estudiantes: sus fortalezas y debilidades en términos de madurez emocional, tomando en cuenta las características derivadas del ciclo evolutivo y otras particularidades. Finalmente, se han proporcionado estrategias que permitan la intervención oportuna, en función a las necesidades y características de cada contexto específico.
\end{abstract}

\title{
Referencias Bibliográficas
}

Colell, J. y Escudé, C. (2003). L’educació emocional. Traç, Revista dels mestres de la Garrotxa, any XIX, num. 37, pp. 8-10. (Original en lengua catalana). En: (http://www.xtec.es/ jcollell/Tra\%E7_6_2003.pdf)

Delors, J. y cols. (1996). La educación encierra un tesoro. Informe a la UNESCO de la Comisión Internacional sobre la educación para el siglo XXI, presidida por Jacques Delors. Madrid : UNESCO / Santillana.

Elías, M.; Tobias, S. y Friedlander, D. (2004).Educar a adolescentes con inteligencia emocional. Barcelona: Debolsillo.

Extremera, N. y Fernández-Berrocal, P. (2004). La importancia de desarrollar la inteligencia emocional en el profesorado. En: Revista Iberoamericana de Educación. Número 33/8. ISSN: 1681-5653 En: (http://www.rieoei.org/deloslectores/465Extremera.pdf)

Galindo, A. (2003). Inteligencia Emocional para jóvenes. Madrid: Prentice Hall Galván Álvarez, A. (2005). Factores emocionales de la educación. Reflexiones acerca de la educación emocional. En: Revista digital "Investigación y Educación”. Número 20. Septiembre del 2005 Vol.3. ISSN 1696-7208. En: (http://www.csicsif.es/andalucia/modules/mod_revistaense/archivos/N_20_2005/emocional.pdf)

Goleman, D. (2000). La inteligencia emocional. Por qué es más importante que el cociente intelectual. Buenos Aires: Javier Vergara Editor.

Goleman, D. y cols. (2002). El líder resonante crea más. El poder de la inteligencia emocional. Barcelona: Editorial Plaza \& Janés.

Goleman, D. (2007). Inteligencia Social. Barcelona: Círculo de Lectores 
Aก̃̃ 4- №广- Dic. 2008

Vivas de Chacón, M. (2004). Las competencias socio-emocionales del docente: Una mirada desde los formadores de los formadores. En: (http://www.uned.es/jutedu/VivasChaconMireya-IJUTE-Comunicacion.PDF)

Para citar este documento, puede utilizar la siguiente referencia:

BUITRON, Sigrid; NAVARRETE, Patricia (2008). "El docente en el desarrollo de la inteligencia emocional: reflexiones y estrategias” [reseña en línea]. Revista Digital de Investigación en Docencia Universitaria (RIDU) Año 4 - $N^{\circ} 1$-Diciembre 2008. [Fecha de consulta: dd/mm/aa]. <

http://beta.upc.edu.pe/calidadeducativa/ridu/2008/ridu5_art5_pn_sb.pdf $>$

\section{Sigrid Buitrón Buitrón}

sbuitron@upc.edu.pe

Psicóloga Clínica de la Pontificia Universidad Católica del Perú, institución en la que ha ejercido la docencia para las facultades de Estudios Generales Letras, y de Letras y Ciencias Humanas (Departamento de Psicología). Facilitadora de la Escuela de Post Grado de la UPC en talleres de Liderazgo para ejecutivos y empresarios, y docente del curso de Liderazgo Personal en la Facultad de Derecho y División de Estudios Profesionales para Ejecutivos (EPE). Actualmente, se encuentra a cargo de la Coordinación de Calidad Educativa EPE de la UPC, siendo responsable de los procesos de capacitación a docentes, talleres de Desarrollo de Competencias para alumnos y consejería psicológica.

\section{Patricia Navarrete Talavera pnavarr@upc.edu.pe}

Licenciada en Psicología por la Pontificia Universidad Católica del Perú. Jefa del Área de Orientación del Departamento de Calidad Educativa de la Universidad Peruana de Ciencias Aplicadas (UPC) desde el año 2007. Psicóloga con experiencia en el campo educativo: consejería psicológica, orientación vocacional, diseño y desarrollo de programas, capacitación y coordinación de equipos docentes, consultorías a instituciones educativas, evaluación de docentes y personal de instituciones educativas, investigación psicoeducativa, y docencia universitaria. Ha sido facilitadora en talleres de desarrollo personal y trabajo en equipo para estudiantes de Estudios Profesionales para Ejecutivos (EPE) y trabajadores de empresas. 